\title{
Perpetual role of brachytherapy in organ-sparing treatment for bladder cancer: a historical review
}

\author{
Elzbieta Van der Steen-Banasik, MD!, Bernard Oosterveld, PhD!', Geert Smits, MD², Els Atema, PhD', \\ Marion Van Gellekom, PhD', Marie Haverkort, MD', Andries Visser, PhDl \\ IRadiotherapiegroep, Arnhem, The Netherlands, ${ }^{2}$ Rijnstate Hospital, Arnhem, The Netherlands
}

\begin{abstract}
Brachytherapy-based radiotherapy has been applied for decades in the curative treatment for solitary, $\leq 5 \mathrm{~cm}$ bladder tumors. This review provides a historical perspective of this organ sparing approach.

A systematic search of the published literature between 1900 and 2019 was performed on the subject of bladder brachytherapy using PubMed, with digitally retrievable articles being supplemented with articles from the personal collection of the authors.

The articles were divided into consecutive time periods, each reflecting the impact of authors on the development of brachytherapy treatment: the time of pioneers, early innovators, modifiers, and recent innovators. Three case-controlled studies comparing brachytherapy-based organ-sparing treatment with cystectomy, demonstrated similarity between the two approaches in terms of disease-free and overall survival, whereas brachytherapy-based approach offered the advantage of at least $80 \%$ chance of bladder preservation. The overview was organized in a chronological order, starting from the evolution of brachytherapy from radium, followed by remote afterloading and dose-rate adjustments, and closing with modern era of high-dose-rate and image-guided brachytherapy. Importantly, we demonstrated how essential and beneficial for the patients is a close collaboration between radiation oncologists and urologists, and how adopting a modern surgical development, i.e. the laparoscopic implantation technique, which later became robot-assisted, contributed to the success of this multidisciplinary brachytherapy treatment.

We concluded that this highly effective brachytherapy method with very limited toxicity deserves more worldwide popularity.

J Contemp Brachytherapy 2020; 12, 6: 618-628 DOI: https://doi.org/10.5114/jcb.2020.101697
\end{abstract}

Key words: bladder cancer, brachytherapy, organ-sparing, high-dose-rate.

\section{Purpose}

Nowadays, in a case of muscle-invasive bladder cancer (MIBC), the patient is offered a radical cystectomy often preceded by neoadjuvant chemotherapy. This treatment is curative, but resulting in a permanent urine deviation, following by a stoma or neo-bladder using parts of the intestine, causing a major negative impact on quality of life [1].

Brachytherapy (BT)-based radiotherapy is one of the alternative bladder-sparing treatment modalities [2], which has been offered to patients with MIBC for decades, but is still one of the best kept secrets in the world of oncology.

Although poor utilized, this treatment has managed to survive through the last century. Consecutive "believers" have introduced various modifications, which have subsequently been taken over by their successors, gradually improving the treatment. After realizing the importance of this treatment evolution, we decided to discuss the subject of brachytherapy focusing on bladder cancer treatment from a historical perspective; therefore, clearly demonstrating its everlasting clinical value.

After the discovery of radium in 1898 by Marie $\mathrm{Cu}$ rie-Sklodowska and Pierre Curie, and before the Nobel Prize was given five years later to Becquerel and the $\mathrm{Cu}$ rie's for their achievements in the field of radioactivity, radiation was used to treat malignant tumors. In fact, the very first radiotherapy was actually brachytherapy: application of radium sources to the tumor area, i.e. irradiation therapy from a short distance.

In the early days, brachytherapy had several significant disadvantages, including a radiation burden for the staff involved and the necessity to isolate the patient for several days. In addition, once the radioactive needles had been inserted, optimization was not possible. However, the introduction of afterloading techniques solved this problem. The radioactive source could be inserted outside of the operating theatre and later with the use of remote afterloading, created a technique, which is com- 
monly practiced today. Further technological advances enabled dose optimization by allowing positions and dwell times of the radioactive source to be predetermined. Finally, the incorporation of modern imaging techniques paved the way for image-guided brachytherapy, with the ability of a 3-dimensional implant and dose reconstructions, in relation to the tumor and surrounding tissues, i.e., organs at risk (OARs). Recently, important essential improvement in surgical technique took place.

\section{Reports from the time of pioneers (see Table 1)}

The first report on treatment with radium sources for bladder cancer that we were able to retrieve was a paper by Barringer [3], in which the development of this treatment in the Memorial Hospital, NY, USA was thoroughly described. In 1915, the first patient was successfully treated using an intravesical method. In June 1919, the Memorial group developed a method of application of radium through the bladder opened suprapubically. Technical aspects of the treatment were precisely described and until 1921, the group treated 142 cases of what they classified as, advanced carcinomas.

In 1942 Herger and Sauer [4] from Buffalo, USA, reported on 267 patients treated between 1930 and 1939, with a 3-year disease-free survival (DFS) of 53\% and 5-year DFS of $37.5 \%$. Based on their experience, brachytherapy indications were established: a solitary tumor $\leq 5 \mathrm{~cm}$, pretreated with Roentgen therapy or electrocoagulation. They also observed that solid tumors not responding to Roentgen irradiation might respond favorably to interstitial radium irradiation. We were astonished to discover that the indications for the treatment did not differ from those applied today. However, the dosimetry aspects of their treatment were underreported, reflecting the pioneering character of bladder brachytherapy during the early days $[3,4,5,6,7,8]$.

\section{Reports from the time of early innovators (see Table 2)}

In 1951, Rotterdam's urologists disappointed by the results of surgical treatment for bladder cancer, consulted the Rotterdam Radiotherapy Institute about radiotherapeutic methods of treatment. Van der Werf-Messing [9] adopted the treatment at a full speed, reporting on a total of 749 patients during the following decades. The importance of Brigit van der Werf-Messing in the development of bladder brachytherapy cannot be emphasized enough. In the successive years, she performed a meticulous analysis of tumor and treatment parameters, including complication rate. She also introduced a method for spatial reconstruction of the implants for the purpose of checking the positions of radium needles and calculating the dose rates at various points (Figure 1). By describing and analyzing the above, she developed a basic knowledge concerning dose-tumor response and normal tissue tolerance. She succeeded in maintaining a close collaboration with the Rotterdam's urologists, which was the backbone of the overall success of the Rotterdam group. Moreover, based on her clinical experience, Van der Werf-Messing established a treatment protocol, which is still applied today. Initially, a low-dose external beam radiotherapy (EBRT), 3 fractions of 3.5 Gy delivered to the small pelvis to prevent scar metastases, pave the way for radium implantation. In the subsequent decades, post-operative EBRT, 30 Gy in 3 weeks was added to the protocol in the case of positive lymph nodes $[10,11,12,13,14,15,16]$. In 1986, Wijnmaalen and Van der Werf-Messing updated their treatment schedule [17]. Based on an observation that a long overall treatment time due to combination therapy, with pre- and post-operative EBRT, resulted in decreased local control (LC), they introduced pre-operative 40 Gy EBRT $(20 \times 2$ Gy, daily). This schedule became a standard for T2 and T3 brachytherapy eligible tumors, and a 5-year disease specific survival (DSS) of $80 \%$ in 90 patients was reported by Van der Werf-Messing and Van Putten in 1989 [18].

The aim of this high-dose external beam irradiation in T2 tumors was not only to prevent scar metastases, but also to reduce the tumor volume and to treat regional lymph nodes. It was followed within a few days by radium implantation of the tumor area.

Interestingly, this treatment was well adopted primarily in The Netherlands, France, and Belgium, but failed to

Table 1. Pioneers

\begin{tabular}{|c|c|c|c|c|}
\hline Author(s) (year) [ref] & Period & $n$ & Survival & Dose \\
\hline Barringer (1921) [3] & since 1915 & 11 intravesicular & max 4 years & - \\
\hline Memorial Hospital, New York, USA & since 1919 & 29 suprapubic & max 20 months & - \\
\hline $\begin{array}{l}\text { Morson (1929) [5] } \\
\text { St. Peter's Hospital, London, UK }\end{array}$ & since 1925 & $23^{a}$ & - & - \\
\hline $\begin{array}{l}\text { Smith (1934) [6] } \\
\text { Radium Institute, London, UK }\end{array}$ & since 1927 & 38 & $20 \%$ & - \\
\hline $\begin{array}{l}\text { Herger and Sauer (1942) [4] } \\
\text { Buffalo }\end{array}$ & 1930-1939 & $267(<5 \mathrm{~cm})$ & $\begin{array}{c}\text { Solid tumors }<5 \mathrm{~cm} \\
\text { 3-year DFS, } 53 \%\end{array}$ & - \\
\hline $\begin{array}{l}\text { Lenz et al. (1946) [7] } \\
\text { New York, USA }\end{array}$ & 1931-1941 & 44 & 5-year DFS, 21\% & 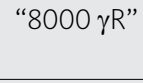 \\
\hline $\begin{array}{l}\text { Barringer (1947) [8] } \\
\text { Memorial Hospital, } \\
\text { New York, USA }\end{array}$ & till 1942 & 255 & 5-year DFS, 37.5\% & - \\
\hline
\end{tabular}

a palliation of hemorrhage, DFS - disease-free survival 


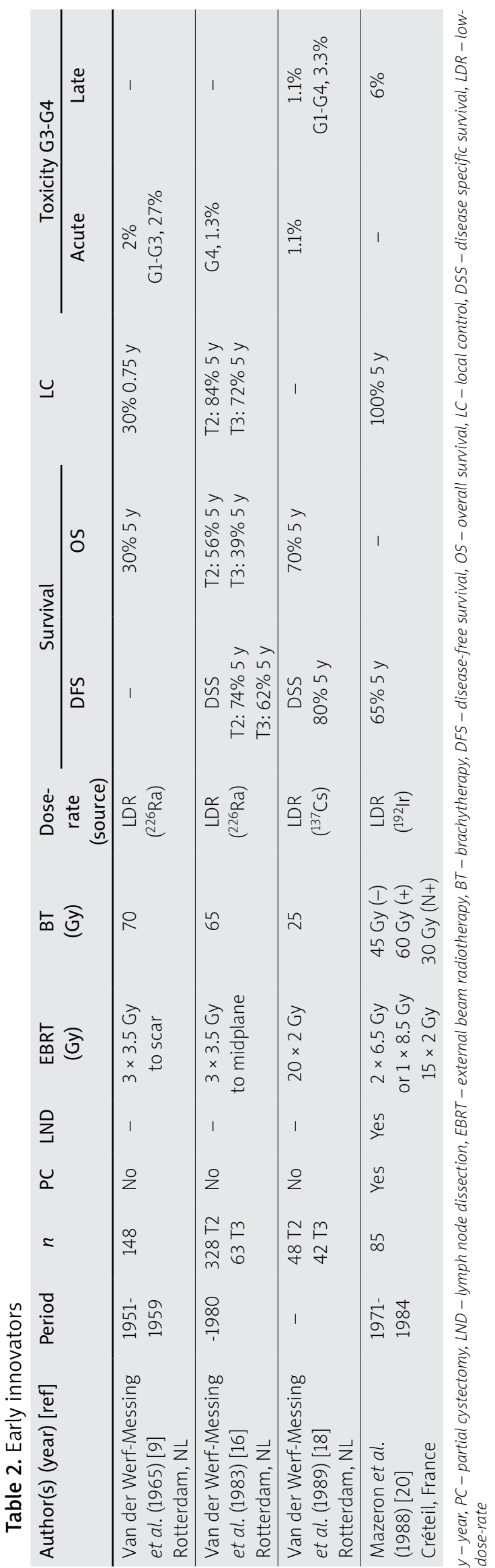

achieve enough of interest outside those countries to be implemented into clinical practice.

French early brachytherapy pioneers reported in French language, causing less accessible communication. Fortunately, in 1985 and 1988, Mazeron et al. [19,20] reported on what we call "the French school". This consisted of a pre-operative short course of EBRT $(2 \times 6.5 \mathrm{~Gy})$, external iliac lymph node dissection, limited partial cystectomy (PC), if necessary combined with ureter re-implantation, and finally implantation of hollow catheters in the margins of partial cystectomy and iridium-192 ( ${ }^{192}$ Ir) wires afterloading. They described a population treated between 1971 and 1984, and the reported results were corresponding with those of Van der Werf-Messing. In addition, they emphasized that they achieved a 5-year LC of $100 \%$ in T2 tumors and strongly advocated for PC whenever possible, depending on the tumor location within the bladder. The undisputable advantage of PC is that the information is obtained about $\mathrm{T}$ stage. In the case of $\mathrm{T} 2$ tumors, they introduced additional post-operative iliac node irradiation.

\section{Reports from the time of modifiers (see Table 3)}

Fortunately, the supporters of the treatment described their results thoroughly. The early innovators were followed by modifiers, who subsequently introduced alterations aimed at improving the treatment. Moreover, they also emphasized the importance of strong collaboration with urologists.

In 1986, a paper by Batterman and Tierie [21] from the Dutch Cancer Institute (NKI) was published reporting on 123 patients treated between 1972-1983. Their modification introduced in 1978 consisted of replacing the short pre-operative irradiation course with 15 fractions of 2 Gy combined with a higher brachytherapy dose. This resulted in a cumulative tumor dose of $70 \mathrm{~Gy}$.

In 1992, De Neve et al. [22] reported retrospectively on a single-institute experience from the Catherina Hospital, Eindhoven, The Netherlands, between 1974 and 1984. Three groups treated with EBRT alone, EBRT with

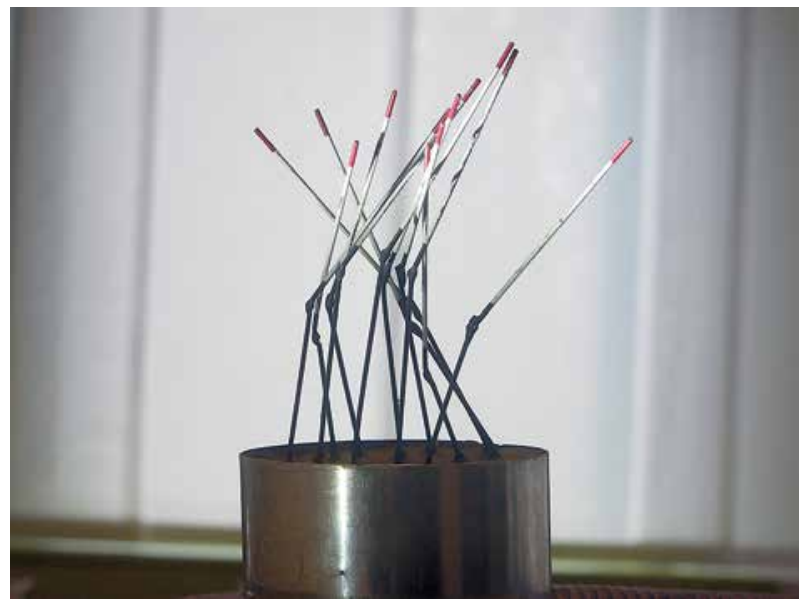

Fig. 1. Spatial reconstruction from the Arnhem collection following guidelines from Van der Werf-Messing. Patient was treated in the 70's 


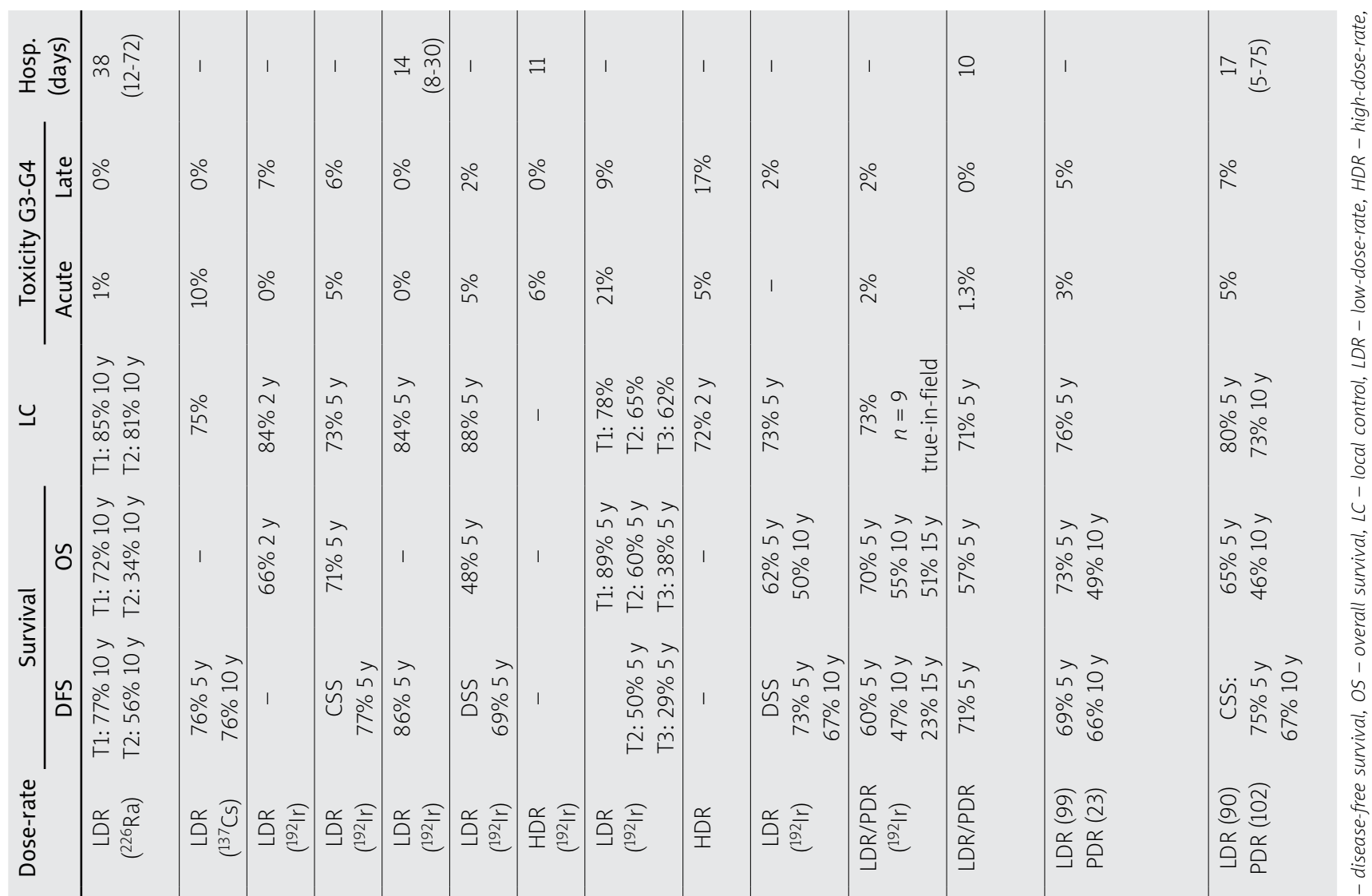

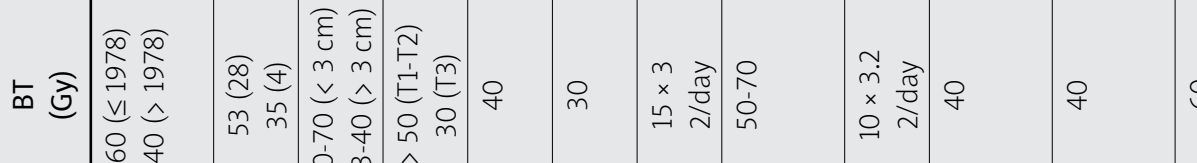

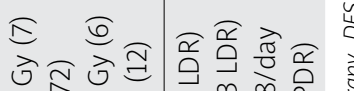

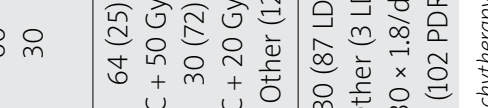

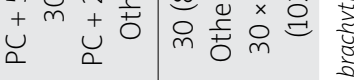

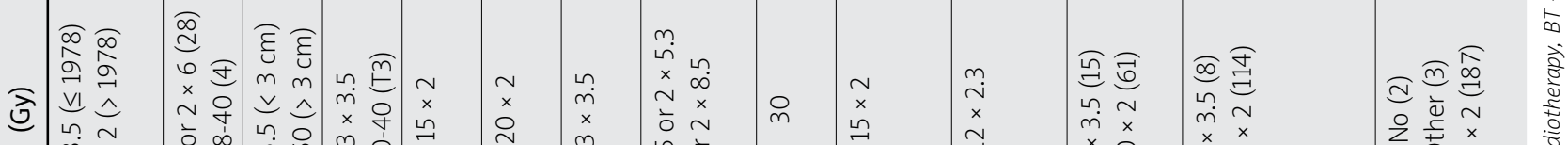

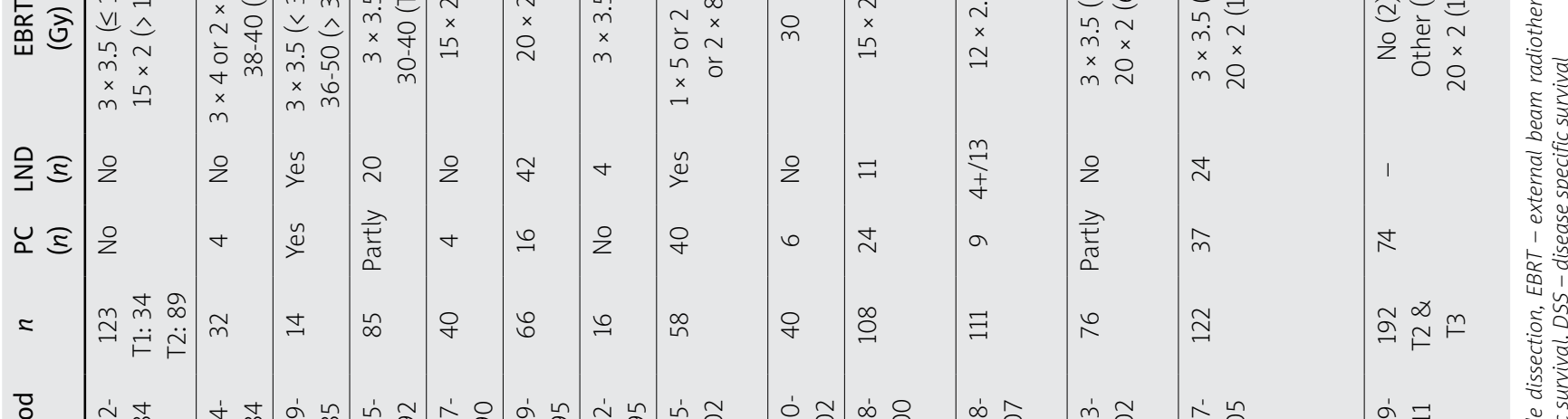

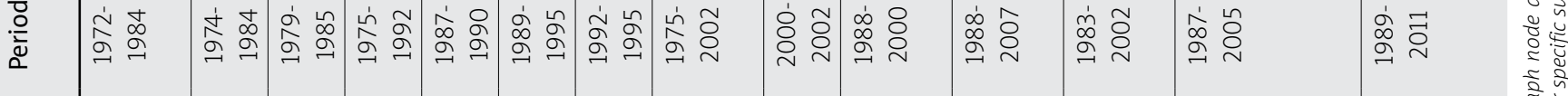

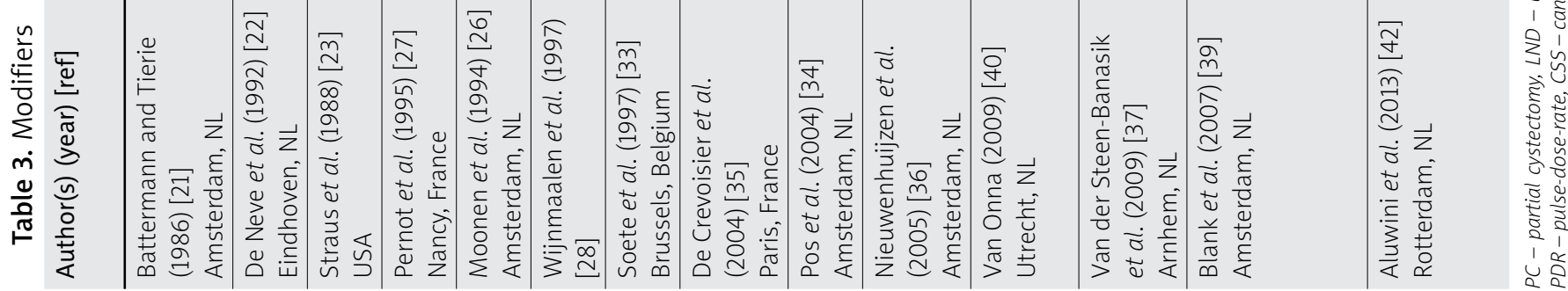




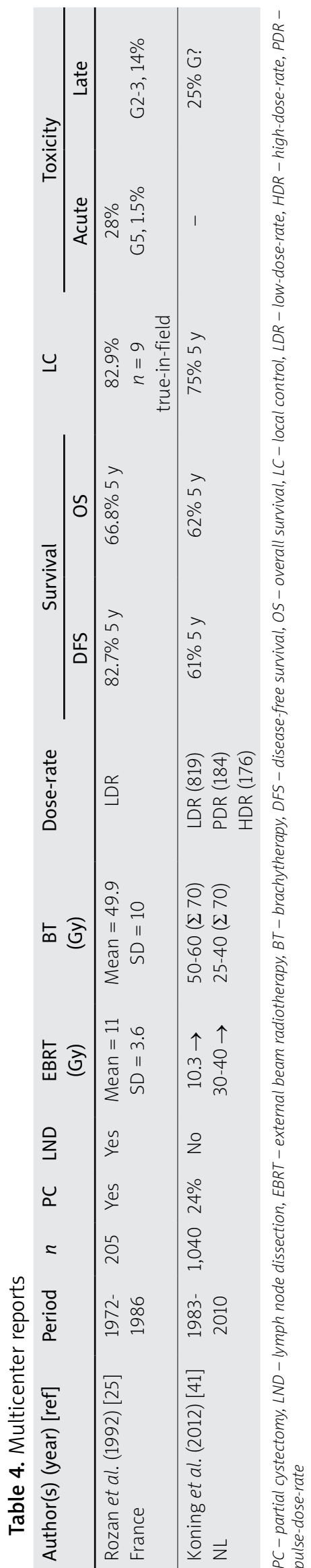

cystectomy, and EBRT with brachytherapy were compared. Out of the 273 patients, 32 received a brachytherapy-based schedule as per the Rotterdam schedule. In this group, a 76\% of 5-year DFS was achieved, superior to the 2 other groups: $50 \%$ and $49 \%$, respectively.

In the meantime, we found that brachytherapy treatment had not been entirely abandoned in the United States. From the post-pioneers' era, we were able to find two reports. In 1988, Straus et al. [23] who advocated in favor of BT, described a population of 14 patients treated in Pennsylvania, with a 2-year actuarial survival of $66 \%$ and LC of $84 \%$. In 1993, Grossman et al. [24] confirmed this advocacy and reported on 7 patients, in whom no in field recurrences occurred.

In the nineties, more single-institution reports followed, sharing their experience either of the French or the Dutch school, and introducing technical improvements, which were gradually implemented over time. Radium and cesium needles were replaced with easy to manage iridium wires, and the introduction of afterloading technique halted the radiation burden for the staff and environment.

The first multicenter study occurred in 1992, when Rozan et al. [25] reported 5-year DFS of $82.7 \%$ (T1, 92.5\%; $\mathrm{T} 2,80.9 \%$; T3a, 62.2\%) in 205 patients, treated in 8 French centers as per the French school. Only 9 true-in field relapses appeared, 2 of these were in the PC group. One of them was originally a multifocal tumor, the other a T3b tumor (Table 4).

In 1994, Moonen et al. [26] described outcomes of 40 patients treated in NKI, Amsterdam, The Netherlands, using ${ }^{192} \mathrm{Ir}$ wires instead of ${ }^{137} \mathrm{Cs}$ needles. They concluded that this modification provided equal results with $86 \%$ DFS, but with significantly less complications due to a new implantation technique, with no radiation exposure for the staff. Instead of radioactive needles, empty catheters were implanted in the bladder wall. The radioactive iridium wires were inserted into the catheters in an isolated environment. The removal of catheters was achieved by a quick and easy withdrawal of the radioactive material, requiring no anesthesia, and causing no discomfort to the patient. In 1996, Pernot et al. [27] described 5-year DFS of $72 \%$ in a population of 85 patients also treated with ${ }^{192} \mathrm{Ir}$, and confirmed the benefits described by the Amsterdam group, and in 1997 also by Rotterdam group [28].

\section{The dose-rate issue}

In the first half of the $20^{\text {th }}$ century, all brachytherapy treatments were applied using low-dose-rate (LDR), a continuous delivery of irradiation due to characteristics of radium, cesium, and iridium, and other available isotopes. After an afterloading standardization presented in 1953 by Henschke [29], it was agreed to keep the doserate typically at $60 \mathrm{cGy} / \mathrm{hr}$. In the 1960's, a high-dose-rate (HDR) regimen was pioneered. Theoretical research on this subject was described in 1991 by Orton [30]. Even though it was received with some hesitation in terms of safety as well as enhanced toxicity and uncertain effectiveness, a clinical research with HDR followed, initially limited to gynecological brachytherapy [31]. The benefit 
of outpatient treatment, short immobilization time, and no lengthy isolation, made the treatment very attractive and psychologically more acceptable. Evidence was gradually generated, primarily for intraluminal BT (intrabronchial, esophageal, and vaginal) and later expanding to all indications. HDR was followed in 1991 by the pulse-doserate (PDR) concept developed by Brenner and Hall [32]. They proposed a remote afterloading technique using a single stepping higher activity radioactive source similar to HDR, but equivalent to LDR, which would replace the complex inventory of sources. The computer-controlled dwell times launched the possibility of a much better dose optimization. At the end, this concept has not become as widely applied as predicted due to several factors, which are beyond the scope of this paper. From a practical point of view, there appeared to be a general preference for fractionated HDR. However, with one exception: bladder cancer.

\section{Yet, there were 2 early reports}

The first one was from Belgium. In 1997, Soete et al. [33] reported on the feasibility of HDR in bladder BT in 16 patients treated between 1992 and 1995. They combined low-dose EBRT with 15 fractions of 3 Gy, 2 fractions a day. Of 15 evaluable patients, 2 developed local recurrences, all experienced mild bladder spasms during the treatment, and there was no symptomatic late toxicity. This was followed by a paper from NKI, in which, Pos et al. [34] described 40 patients treated with their usual schedule of 30 Gy EBRT, followed by HDR, 10 fractions of 3.2 Gy, applied twice daily. They compared the outcomes with those of their historical LDR group, and concluded that DFS was inferior with 2 -year LC of $72 \%$ vs. $88 \%$, and unacceptable toxicity in 5 of 40 patients, with G3 late toxicity vs. 2 of 84 LDR patients. The Amsterdam report was taken as a serious warning and for quite a few years, HDR was considered to be a non-beneficial modification with consequences, which might be fatal for the future of bladder brachytherapy. Further research on this topic was clearly needed, as we will demonstrate further in this paper.

At the beginning of the $21^{\text {st }}$ century, the utilization of brachytherapy in bladder cancer appeared to decline and was gradually replaced by chemoradiation, which offered less logistic problems, no need for operation theatre facilities, no hospitalization, and straight forward treatment in countries where chemotherapy and radiotherapy were administered by the same specialist. The discouraging NKI report concerning HDR utilization might have played a role in the declining utilization, simply because several departments had replaced LDR with HDR afterloaders.

Fortunately, some supporters maintained the modality in their treatment plans and in the first decade of the $21^{\text {st }}$ century, only occasional reports appeared, generally limited to The Netherlands, with the exception of one French publication. In 2004, de Crevoisier et al. [35] reported the results of 25 years' experience of the Institute of Gustave-Roussy in Paris where from 1975-2002, 58 patients were treated conforming the French school, show- ing 5 -year DFS of $50 \%$ in T2 tumors and 5-year probability of bladder preservation of $65 \%$.

In 2005 and in 2009, two reports were published comparing the outcomes of brachytherapy-based treatment with those of radical cystectomy. Nieuwenhuijzen et al. [36] compared T1/T2 patients treated in NKI either with a brachytherapy-based schedule $(n=108)$ or with radical cystectomy (RC) $(n=77)$. These two described groups were not entirely comparable, which was a limitation of this study. While the BT group fulfilled the usual criteria, the surgery group included patients with multifocal tumors. On the other hand, the RC group included less of T2 tumors with unknown diameter. Age-adjusted $5 / 10$-year disease specific survival (DSS) was $75 / 70 \%$ vs. $66 / 66 \%$, and they modestly concluded that regarding survival, these results did not provide evidence against the use of BT in this selected population. They also pointed out that $90 \%$ of the long-term survivors treated with BT preserved their bladder.

In 2009, Van der Steen-Banasik et al. [37] reported on their retrospective case control study of two populations with solitary, T2 tumors eligible for brachytherapy-based treatment (BT). One group consisted of 65 patients treated by cystectomy in Radboud University Medical Centre, Nijmegen, where BT was not a part of the department's policy, and the other group involved 77 patients in Rijnstate Hospital Arnhem treated with BT. Within the statistical uncertainty, there was no difference in 5- and 10-year DFS. The evident difference could be found both acute and late in toxicity, favoring the radiotherapy group. The final benefit for $70 \%$ of the BT patients was preservation of a well-functioning bladder. The technique was already described in detail by Van der Steen-Banasik et al. in 2002 [38].

In 2007, Blank et al. [39] compared the results of LDR BT schedule $(n=99)$ vs. biologically equivalent PDR BT schedule (104 cGy/pulse, every $2.2 \mathrm{~h}$, to a total cumulative tumor dose of approximately $70 \mathrm{~Gy})(n=23)$ used from 1987 till 2005 in one of the three collaborating institutes in Amsterdam. EBRT was either 10.5 Gy in 3 fractions, followed by 64 Gy BT, or 40 Gy in 20 fractions, followed by 30 Gy BT. No differences were observed in terms of outcomes or toxicities.

This observation was confirmed by the Utrecht group in 2009, where Van Onna et al. [40] reported on 111 patients treated until 2003 with LDR and with a PDR schedule thereafter, concluding that the regimes were entirely equivalent.

A second retrospective multi-center study performed by Koning et al. [41] followed in 2012, reporting on 1,040 patients treated in 10 Dutch centers between 1983 and 2010 (Table 4). Once again, the evident efficacy of the treatment was demonstrated, showing 61\% 5-year DFS and $75 \%$ 5-year LC, and the reported toxicity was mild. The brachytherapy was performed either with LDR or PDR, and a small minority of 15 patients were treated with HDR. There was not much difference between the outcomes of the institutes.

In 2013, Aluwini et al. [42] described 192 patients treated in Rotterdam, The Netherlands, between 1989 and 2011. Initially, 90 patients were treated with LDR and 


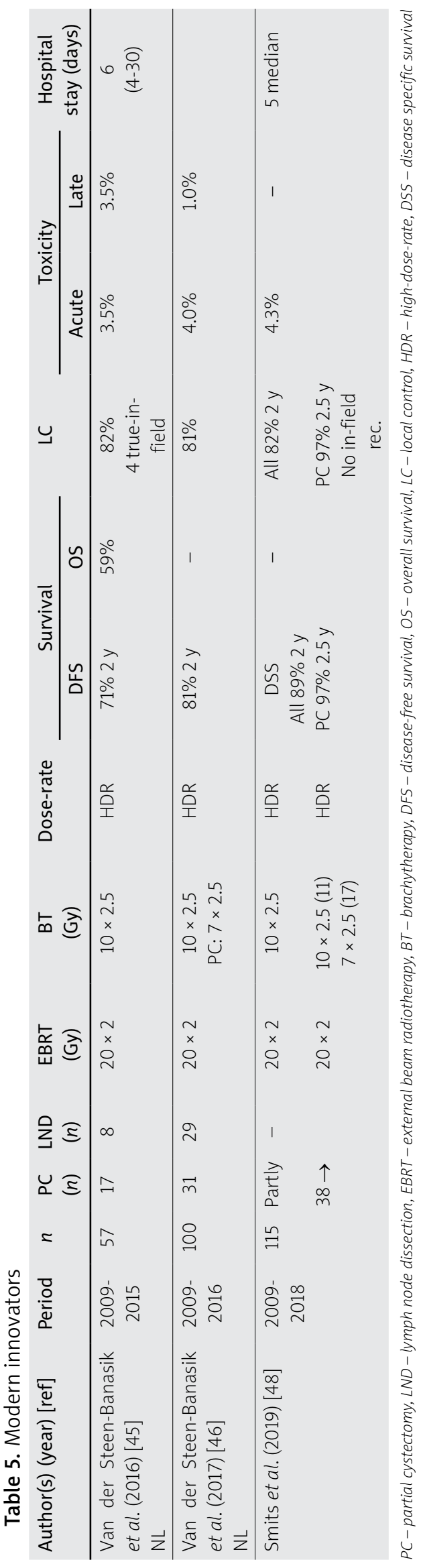

from 1992 with PDR $(n=102)$, with a slightly different schedule from the one used in Amsterdam, i.e., $1 \mathrm{~Gy} \mathrm{ev-}$ ery 3 hours continuously applied, which was still considered to be radiobiologically equivalent.

\section{Reports from the time of modern innovators (see Table 5)}

In the second decade of the $21^{\text {st }}$ century, new innovations were introduced by the Arnhem group in 2009 [4348]. The first innovation was replacing the classical open suprapubic approach using Pfannenstil incision by a minimally invasive approach. This approach had already been dominating the surgical field, but had never been applied to insert interstitial catheters. This was achieved by a simultaneous application of cystoscope and laparoscope, and obtaining a bladder view from inside and outside to facilitate interstitial catheter placement within the bladder wall, thus avoiding intraluminal bladder perforations (Figure 2). Within one year, this approach was replaced by a robot-assisted technique [43].

The radiotherapeutic part of the procedure was described in 2014 by Nap-Van Klinken et al. [44], who concluded that the quality of the implant remained guaranteed and problems with catheter accessibility were greatly reduced.

Shortly thereafter, in 2016, the first report on the results of the treatment using this method was published by Van der Steen-Banasik et al. [45]. They described their 5 years of experience, using a laparoscope and simultaneously changing the PDR into a HDR regimen, specifically calculated for the purpose of bladder brachytherapy, which was their second innovation. The HDR schedule was calculated using BED and EQD, and differed from the scheme described by Pos et al. [34]. They combined EBRT 40 Gy in 20 fractions with BT 25 Gy in 10 fractions (3 fractions a day). In the population of 57 patients, using incidence competing risk analysis, the 2-year overall survival (OS), DSS, and LC were $59 \%, 87 \%$, and $82 \%$, respectively. Their HDR scheme proved to be safe, with only mild toxicity, and hospitalization time of the patients was halved [45].

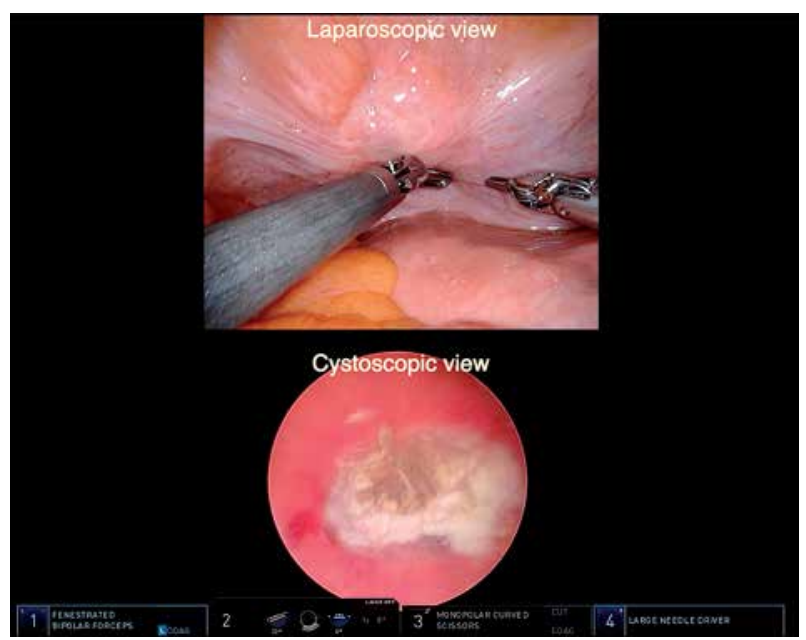

Fig. 2. Laparoscopic and cystoscopic views during minimally invasive Arnhem procedure 

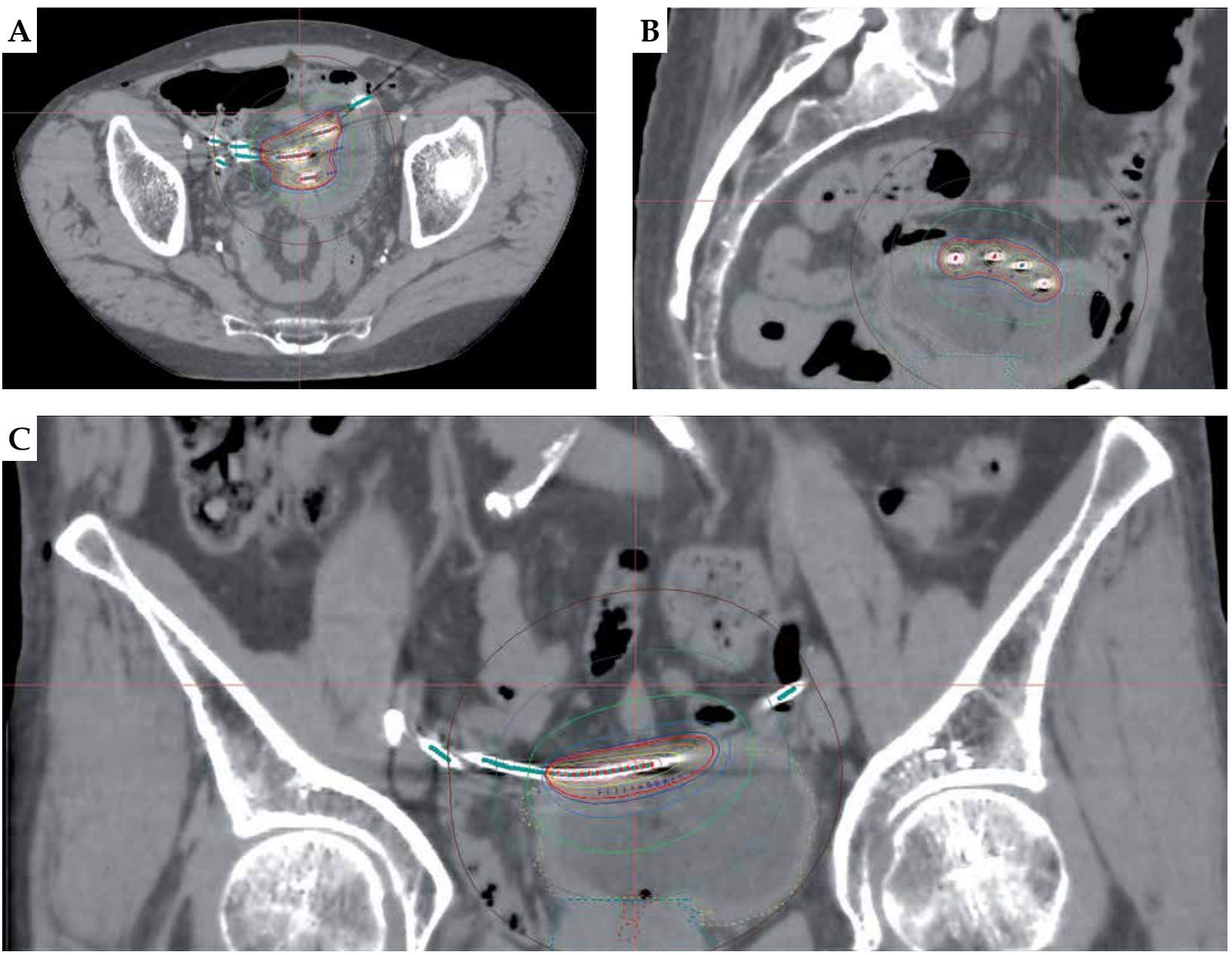

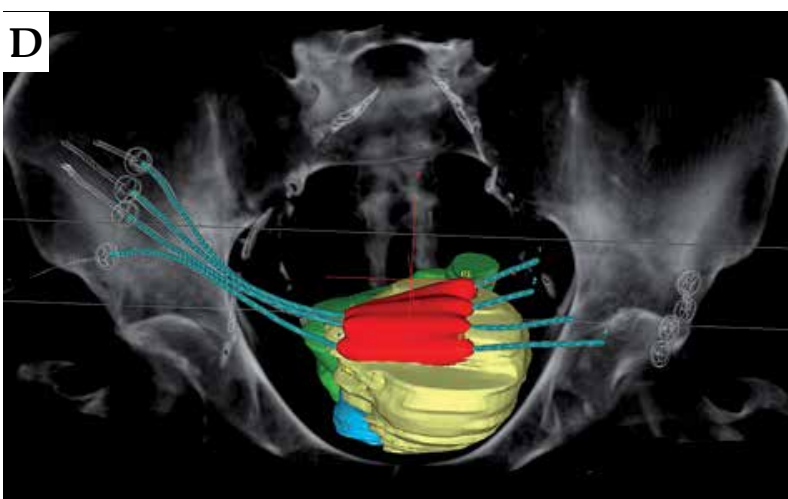

Since 2016, they decided to de-escalate the brachytherapy dose to 17.5 Gy in 7 fractions, if PC was performed [46].

In 2018, Bus et al. [47] reported on catheter position verification, with the QA system consequently applied by the Arnhem group, following the modern image-guided brachytherapy (IGBT) development (Figure 3).

Recently, the Arnhem group described the outcomes of a partial cystectomy followed by brachytherapy (17.5 Gy) after EBRT $(49 \%$ pT0, $11 \%$ pT1, and $40 \% \geq$ pT2), showing in the first 38 consecutive cases no in-field local recurrences, 97\% cancer-specific survival, 97\% loco-regional control, and $94 \%$ no evidence of disease, during a median follow-up of 2.5 year (range, 4 months to 8 years) [48].
Fig. 3. Implantation with 4 catheters, imaged and planned on CT. Transversal (A), sagittal (B), coronal (C), and 3D (D) views. Light blue dots: reconstructed catheters. Red dots: dwell positions. Red line: $100 \%$ isodose contour. On the $3 \mathrm{D}$ reconstruction: organs involved are bladder (yellow), sigmoid (green), and rectum (blue). The 100\% isodose volume is red. (3D, three-dimensional)

Both the robot-assisted procedure and the use of HDR were adopted at NKI, Amsterdam, where in 2018, Bosschieter [49] described the implantation procedure pioneered in Arnhem. The method was also adopted in Lisbon, where Mascarenas [50] showed feasibility of the treatment.

In a more recent paper, from October 2019, Voskuilen et al. [51] compared the Amsterdam treatment outcomes of 60 RC vs. 259 BT-based procedures in T2 patients treated in between 1988 and 2016. They confirmed the results already published in 2009 [37] and concluded that bladder-preserving therapy with BT may be considered a reasonable treatment option in highly selected patients with 
solitary cT1G3-T2N0M0 tumors, with 10-year DSS of 67\% after BT vs. $65 \%$ after RC with $84 \%$ bladder preservation and less high-grade complications.

\section{Discussion}

Within the oncology community, there is a growing interest in the patient's quality of life. Organ sparing is certainly an undisputable part of this philosophy and can be achieved thorough an interdisciplinary approach. Although cystectomy is a standard treatment in MIBC, there has always been interest in a bladder-sparing procedure, whenever feasible. Attempts to perform a randomized study comparing cystectomy with bladder-sparing approaches have never succeeded.

Worldwide, there are 3 approaches towards organsparing management in MIBC: 1) Surgical: partial cystectomy (PC); 2) Combined modality therapy (CMT), including trans urethral resection of the bladder (TURB), chemotherapy (neoadjuvant, concomitant, or both), and EBRT; and 3) The approach including TURB, EBRT, and BT.

The first approach, PC, is considered in a case of solitary tumor that can be resected with a 1-2 cm tumor-free margin without carcinoma in situ (CIS) with lymph node dissection (LND) as a scheduled part of the procedure. Single-institution series from the MD Anderson Cancer and Memorial Sloan Kettering Cancer Center, reported 5 -year DFS ranging from $39 \%$ to $67 \%[52,53]$. Using the SEERS-9 database, Capitanino et al. [54] demonstrated that compared to radical cystectomy (RC), PC does not compromise survival for selected patients.

The second approach, CMT, was developed at the Massachusetts General Hospital and adopted in the Radiation Therapy Oncology Group (RTOG) $[55,56]$. In 2014, Mak et al. [57] reported a pooled analysis of long-term outcomes of 468 patients, enrolled into 6 RTOG studies, treated from 1988 till 2007. A 5-year DSS rate was 71\% for the whole group and $74 \%$ for T2 tumors, which were $60.6 \%$ of the population. In contrast to a brachytherapy-based approach, CMT does not require specific tumor selection.

The third approach, TURB, EBRT, and BT, reported in the present paper, is considered in a case of solitary $\mathrm{T} 2 \mathrm{tu}$ mor $<5 \mathrm{~cm}$, without CIS. The treatment can be combined with PC; however, a tumor-free margin is not mandatory. The French school includes PC and LND, while in the Dutch school, the inclusion of PC and LND is performed only if indicated.

There are only two multicenter studies, both retrospective. The Dutch population of 1,040 patients demonstrated 5-year LC of 75\% and 5-year DFS of $61 \%$ [41], and this is comparable to the French multicenter survey of 205 patients reported by Rozan et al. [25]. All other studies are single-institute reports.

These results appear to be favorable or comparable to both PC alone and CMT, and the treatment seems less complicated in comparison to CMT, keeping in mind that in the brachytherapy population, selected tumor stages are not higher than $\mathrm{T} 2$.

Regrettably, to our knowledge, only two national guidelines (Dutch [58] and French [59]) mention brachytherapy as a modality to be considered in the treatment of a solitary MIBC, despite the GEC-ESTRO recommendations published in 2017 [60]. Besides, there are data confirming that robot-assisted brachytherapy is the most cost-effective treatment strategy, and should be the preferred strategy for a selected group of MIBC.

\section{Conclusion and future perspectives}

Bladder-preserving therapy using brachytherapy has a long history, nearly as long as the history of radiotherapy and its development follows a technical transformation from radium implantation, through (remote) afterloading, to HDR and IGBT.

If applied, according to the usual selection criteria, the treatment is highly effective, providing a high curative rate, and is at least comparable with cystectomy, with the advantage of very limited toxicity, allowing for the preservation of the bladder. A close collaboration between the urologists and radiation oncologists is the fundamental backbone for this multidisciplinary approach, and this brachytherapy-based procedure is an excellent example of the importance of close interdisciplinary partnership, resulting in clinical benefit for patients.

The role of lymph node dissection has not yet been established and remarkably, when comparing the results of the Dutch and French schools, there seems to be no outcome difference in terms of survival, despite the different protocols for lymph node management. The combination with partial cystectomy (if applicable) provides the optimal chance of local control. A multi-institutional registration study could provide with more evidence about the factors associated with optimal outcomes, and would also deliver an opportunity to share the results, to introduce the treatment more widely and hopefully, to answer the question of significance of lymph node dissection in this patients' population.

The feasibility of extending this approach to more advanced tumors in combination with chemo- or immunotherapy could also be investigated.

Organ-sparing bladder cancer treatment including brachytherapy definitely deserves a wider popularity and broader use in clinical practice for the benefit of patients.

\section{Panel}

A systematic search of the published literature on brachytherapy treatment for bladder cancer between 1900 and 2019 was completed in the PubMed database. We decided to collect papers from the very onset of brachytherapy, because this gives an interesting and even surprising historic perspectives.

The PubMed database was searched for articles in English language using the terms, such as "bladder carcinoma" (or synonyms neoplasm, tumor, cancer) combined with "brachytherapy" (or Radium, Iridium, Cesium, implantation). From the initial search reviews, meta analyses, case reports, and book chapters were excluded. By reading the title and abstract of a publication, malignancies other than bladder cancer, treatments other than 
brachytherapy, and duplications were excluded. The article search was extended by verifying the references of the relevant papers. Unfortunately, some of the older important articles could not be retrieved by our search system (collection of the Radboud University Medical Center (UMC), Nijmegen). Only digitally retrievable articles and papers from personal collections of the authors were used. The reference list was generated based on originality and relevance to the scope of this present review.

\section{Disclosure}

The authors report no conflict of interest.

\section{References}

1. Winters BR, Wright JL, Holt SK et al. Health related quality of life following radical cystectomy: comparative analysis from the medicare health outcomes survey. J Urol 2018; 199: 669-675.

2. Vashistha V, Wang H, Mazzone A et al. Radical cystectomy compared to combined modality treatment for muscle-invasive bladder cancer: A systematic review and meta-analysis. Int J Radiat Oncol Biol Phys 2017; 97: 1002-1020.

3. Barringer BS. Radium treatment of carcinoma of the bladder. Ann Surg 1921; 74: 751-767.

4. Herger C, Sauer HR. Radium treatment of cancer of bladder. Report of 267 cases. Am J Roentgenol 1942; 47: 909-915.

5. Morson AC. Observations on twenty-three cases of carcinoma of the bladder treated by radium. Br Med J 1929; 8: 1038-1039.

6. Smith AJ. The use of radium in carcinoma of the bladder. Br Med J 1934; 2: 584-587.

7. Lenz M, Cahill GF. The treatment of cancer of the bladder by radium needles. Am J Roentgenol Radium Ther 1947; 58: 486-492.

8. Barringer BS. Twenty-five years of radon treatment of cancer of the bladder. J Am Med Assoc 1947; 135: 616-618.

9. Van der Werf-Messing B. Treatment of carcinoma of the bladder with radium. Clin Radiol 1965; 16: 16-26.

10. Van der Werf-Messing B. Carcinoma of the bladder treated by suprapubic radium implants: The value of additional external irradiation. Eur J Cancer 1969; 5: 277-285.

11. Van der Werf-Messing B. Carcinoma of the bladder treated with intracavitary application of cobalt- 60 beads. Clin Radiol 1971; 22: 101-103.

12. Van der Werf-Messing BH. Cancer of the urinary bladder treated by interstitial radium implant. Int J Radiat Oncol Biol Phys 1978; 4: 373-378.

13. Van der Werf-Messing B, Star WM, Menon RS. T3NXM0 carcinoma of the urinary bladder treated by the combination of radium implant and external irradiation. A preliminary report. Int J Radiat Oncol Biol Phys 1980; 6: 1723-1725.

14. Van Der Werf-messing B, Hop WCJ. Carcinoma of the urinary bladder (category T1NXM0) treated either by radium implant or by transurethral resection only. Int J Radiat Oncol Biol Phys 1981; 7: 299-303.

15. Van der Werf-Messing B, Menon RS, Hop WCJ. Carcinoma of the urinary bladder category T3NXM0 treated by the combination of radium implant and external irradiation: second report. Int J Radiat Oncol Biol Phys 1983; 9: 177-180.

16. van der Werf-Messing B, Menon RS, Hop WCJ. Cancer of the urinary bladder category T2, T3, (NXM0) treated by interstitial radium implant: second report. Int J Radiat Oncol Biol Phys 1983; 9: 481-485.

17. Wijnmaalen A, Van der Werf-Messing BH. Factors influencing the prognosis in bladder cancer. Int J Radiat Oncol Biol Phys 1986; 12: 559-565.
18. Van der Werf-Messing BH, Van Putten WL. Carcinoma of the urinary bladder category T2,3NXM0 treated by 40 Gy external irradiation followed by cesium137 implant at reduced dose (50\%). Int J Radiat Oncol Biol Phys 1989; 16: 369-371.

19. Mazeron JJ, Marinello G, Leung S et al. Treatment of bladder tumors by iridium 192 implantation. The Créteil technique. Radiother Oncol 1985; 4: 111-119.

20. Mazeron JJ, Crook J, Chopin D et al. Conservative treatment of bladder carcinoma by partial cystectomy and interstitial iridium 192. Int J Radiat Oncol Biol Phys 1988; 15: 1323-1330.

21. Battermann JJ, Tierie AH. Results of implantation for T1 and T2 bladder tumours. Radiother Oncol 1986; 5: 85-90.

22. De Neve W, Lybeert ML, Goor C et al. T1 and T2 carcinoma of the urinary bladder: Long term result with external, preoperative, or interstitial radiotherapy. Int J Radiat Oncol Biol Phys 1992; 23: 299-304.

23. Straus KL, Littman P, Wein AJ et al. Treatment of bladder cancer with interstitial iridium-192 implantation and external beam irradiation. Int J Radiat Oncol Biol Phys 1988; 14: 265-271.

24. Grossman HB, Sandler HM, Perez-Tamayo C. Treatment of T3a bladder cancer with iridium implantation. Urology 1993; 41: 217-220.

25. Rozan R, Albuisson E, Donnarieix D et al. Interstitial iridium-192 for bladder cancer (A multicentric survey: 205 patients). Int J Radiat Oncol Biol Phys 1992; 24: 469-477.

26. Moonen LMF, Horenblas S, Van der Voet JCM et al. Bladder conservation in selected T1G3 and muscle-invasive T2-T3a bladder carcinoma using combination therapy of surgery and iridium-192 implantation. Br J Urol 1994; 74: 322-327.

27. Pernot M, Hubert J, Guillemin F et al. Combined surgery and brachytherapy in the treatment of some cancers of the bladder (partial cystectomy and interstitial iridium-192). Radiother Oncol 1996; 38: 115-120.

28. Wijnmaalen A, Helle PA, Koper PC et al. Muscle invasive bladder cancer treated by transurethral resection, followed by external beam radiation and interstitial iridium-192. Int J Radiat Oncol Biol Phys 1997; 39: 1043-1052.

29. Henschke UK, James AG, Myers WG. Radiogold seeds for cancer therapy. Nucleonics 1953; 11: 46-48.

30. Orton CG, Seyedsadr M, Somnay A. Comparison of high and low dose rate remote afterloading for cervix cancer and the importance of fractionation. Int J Radiat Oncol Biol Phys 1991; 21: $1425-1434$.

31. Shigematsu Y, Nishiyama K, Masaki N et al. Treatment of carcinoma of the uterine cervix by remotely controlled afterloading intracavitary radiotherapy with high-dose rate: a comparative study with a low-dose rate system. Int J Radiat Oncol Biol Phys 1983; 9: 351-356.

32. Brenner DJ, Hall EJ. Conditions for the equivalence of continuous to pulsed low dose rate brachytherapy. Int J Radiat Oncol Biol Phys 1991; 20: 181-190.

33. Soete G, Coen V, Verellen D et al. A feasibility study of high dose rate brachytherapy in solitary urinary bladder cancer. Int J Radiat Oncol Biol Phys 1997; 38: 743-747.

34. Pos FJ, Horenblas S, Lebesque J et al. Low-dose-rate brachytherapy is superior to high-dose-rate brachytherapy for bladder cancer. Int J Radiat Oncol Biol Phys 2004; 59: 696-705.

35. De Crevoisier R, Ammor A, Court B et al. Bladder-conserving surgery and interstitial brachytherapy for lymph node negative transitional cell carcinoma of the urinary bladder: Results of a 28-year single institution experience. Radiother Oncol 2004; 72: 147-157.

36. Nieuwenhuijzen JA, Pos F, Moonen LMF et al. Survival after bladder-preservation with brachytherapy versus radical cystectomy; a single institution experience. Eur Urol 2005; 48: 239-245.

37. van der Steen-Banasik E, Ploeg M, Witjes JA et al. Brachytherapy versus cystectomy in solitary bladder cancer: A case con- 
trol, multicentre, East-Netherlands study. Radiother Oncol 2009; 93: 352-357.

38. Van der Steen-Banasik EM, Visser AG, Reinders JG et al. Saving bladders with brachytherapy: Implantation technique and results. Int J Radiat Oncol Biol Phys 2002; 53: 622-629.

39. Blank LECM, Koedooder K, van Os R et al. Results of bladder-conserving treatment, consisting of brachytherapy combined with limited surgery and external beam radiotherapy, for patients with solitary T1-T3 bladder tumors less than $5 \mathrm{~cm}$ in diameter. Int J Radiat Oncol Biol Phys 2007; 69: 454-458.

40. van Onna IEW, Oddens JR, Kok ET et al. External beam radiation therapy followed by interstitial radiotherapy with iridium-192 for solitary bladder tumours: results of 111 treated patients. Eur Urol 2009; 56: 113-122.

41. Koning CCE, Blank LECM, Koedooder C et al. Brachytherapy after external beam radiotherapy and limited surgery preserves bladders for patients with solitary pT1-pT3 bladder tumors. Ann Oncol 2012; 23: 2948-2953.

42. Aluwini S, Van Rooij PHE, Kirkels WJ et al. Bladder function preservation with brachytherapy, external beam radiation therapy, and limited surger in bladder cancer patients: Longterm results. Int J Radiat Oncol Biol Phys 2014; 88: 611-617.

43. Smits GAHJ, Wijburg CJ, van der Steen-Banasik E. Robot assisted laparascopic brachytherapy catheter implantation in bladder cancer: introduction of a new technique. Eur Urol Suppl 2011; 10: 556.

44. Nap-van Klinken AM, Bus SJEA, Janssen TG et al. EP-1944: The quality of the laparoscopically performed interstitial bladder implantation. Radiother Oncol 2014; 111: S338.

45. van der Steen-Banasik EM, Smits GAHJ, Oosterveld BJ et al. The Curie-Da Vinci connection: 5-years' experience with laparoscopic (robot-assisted) implantation for high-doserate brachytherapy of solitary T2 bladder tumors. Int J Radiat Oncol Biol Phys 2016; 95: 1439-1442.

46. van der Steen-Banasik E, Oosterveld B, Wijburg C et al. 100 Patients' experience with brachytherapy in bladder implantations performed laparoscopically in solitary bladder tumors. Int J Radiat Oncol Biol Phys 2017; 99: E270.

47. Bus SJEA, Leus NG, Oosterveld BJ et al. An evaluation of our experience in position verification of catheters used for interstitial high-dose-rate brachytherapy of solitary bladder tumors. Brachytherapy 2018; 17: 24-30.

48. Smits GAHJ, Van der Steen-Banasik E, Oosterveld B et al. Minimal invasive surgery and brachytherapy as bladder preserving multimodality treatment in selected solitary MIBC. Eur Urol Suppl 2019; 18: e814.

49. Bosschieter J, Vis AN, van der Poel HG et al. Robot-assisted laparoscopic implantation of brachytherapy catheters in bladder cancer. Eur Urol 2018; 74: 369-375.

50. Mascarenhas F, Maes K, Marques F et al. Robot-assisted brachytherapy of the bladder with long distance support using video conferencing. J Contemp Brachytherapy 2017; 9: 375-382.

51. Voskuilen CS, Bosschieter J, van Werkhoven E et al. Longterm survival and complications following bladder-preserving brachytherapy in patients with cT1-T2 bladder cancer. Radiother Oncol 2019; 141: 130-136.

52. Kassouf W, Swanson D, Kamat AM et al. Partial cystectomy for muscle invasive urothelial carcinoma of the bladder: a contemporary review of the M. D. Anderson Cancer Center experience. J Urol 2006; 175: 2058-2062.

53. Holzbeierlein JM, Lopez-Corona E, Bochner BH et al. Partial cystectomy: a contemporary review of the Memorial Sloan-Kettering Cancer Center experience and recommendations for patient selection. J Urol 2004; 172: 878-881.

54. Capitanio U, Isbarn H, Shariat SF et al. Partial cystectomy does not undermine cancer control in appropriately selected patients with urothelial carcinoma of the bladder: a population-based matched analysis. Urology 2009; 74: 858-864.

55. Shipley WU, Winter KA, Kaufman DS et al. Phase III trial of neoadjuvant chemotherapy in patients with invasive bladder cancer treated with selective bladder preservation by combined radiation therapy and chemotherapy: initial results of Radiation Therapy Oncology Group 89-03. J Clin Oncol 1998; 16: 3576-3583.

56. Shipley WU, Zietman AL, Kaufman DS et al. Selective bladder preservation by trimodality therapy for patients with muscularis propria-invasive bladder cancer and who are cystectomy candidates - The Massachusetts General Hospital and Radiation Therapy Oncology Group experiences. Semin Radiat Oncol 2005; 15: 36-41.

57. Mak RH, Hunt D, Shipley WU et al. Long-term outcomes in patients with muscle-invasive bladder cancer after selective bladder-preserving combined-modality therapy: a pooled analysis of Radiation Therapy Oncology Group protocols 8802, 8903, 9506, 9706, 9906, and 0233. J Clin Oncol 2014; 32: 3801-3809.

58. Richtlijn Blaascarcinoom Nederlandstalige samenvatting van de EAU guidelines on bladder cancer. www.nvu.nl.

59. D’Urologie AF. Partie B. Chapitre V. Alternatives thérapeutiques à la cystectomie totale pour une tumeur infiltrante de vessie. D. Les alternatives conservatrices du réservoir vésical d'indications plus rares. https://www.urofrance.org/ base-bibliographique/ partie-b-chapitre-v-alternatives-therapeutiques-la-cystectomie-totale-pour-22003.

60. Pieters BR, Van der Steen-Banasik E, Smits GA et al. GECESTRO/ACROP recommendations for performing bladder-sparing treatment with brachytherapy for muscle-invasive bladder carcinoma. Radiother Oncol 2017; 122: 340-346. 